\title{
Candidate Molecules and ki-67/MIB1 as Novel Diagnostic Biomarker for Human Uterine Mesenchymal Tumors
}

\author{
Takuma Hayashi $^{1,2}$, Akiko Horiuchi ${ }^{3}$, Nobuo Yaegashi ${ }^{4}$, Susumu Tonegawa $^{5}$, Ikuo Konishi ${ }^{2}$ \\ ${ }^{1}$ Department of Immunology and Infectious Disease, Shinshu University School of Medicine, \\ Matsumoto, Nagano, Japan \\ ${ }^{2}$ Department of Obstetrics and Gynecology, Kyoto University Graduate School of Medicine, \\ Kyoto-city, Kyoto, Japan \\ ${ }^{2}$ Promoting Business using Advanced Technology, Japan Science and Technology Agency (JST), \\ Chiyoda-ku, Tokyo, Japan \\ ${ }^{3}$ Horiuchi Ladies Clinic, Matsumoto, Nagano 390-0821, Japan \\ ${ }^{4}$ Department of Obstetrics and Gynecology, Tohoku University Graduate School of Medicine, Miyagi Japan \\ ${ }^{5}$ Picower Institution and Department of Biology, Massachusetts Institute of Technology, \\ Cambridge, MA 02139-4307 USA \\ Email: yoyoyo224@hotmail.com
}

Received April, 2013

\begin{abstract}
Human uterine leiomyosarcoma (LMS) develops more often in the muscle tissue layer of the uterine body than in the uterine cervix. The development of gynecologic tumors is often correlated with female hormone secretion; however, the development of uterine LMS is not substantially correlated with hormonal conditions, and the risk factors are not yet known. Importantly, a diagnostic-biomarker, which distinguishes malignant uterine LMS from benign tumor leiomyoma (LMA), is yet to be established. Accordingly, it is necessary to analyze risk factors associated with uterine LMS, to establish a clinical treatment method. Protea some $\beta$-ring subunit LMP2/ $\beta 1$ i-deficient mice spontaneously develop uterine LMS, with a disease prevalence of $\sim 40 \%$ by 14 months of age. We found LMP2/ $\beta 1$ i expression to be absent in human uterine LMS, but present in human LMA. Therefore, defective-LMP2/31i expression may be one of the risk factors for human uterine LMS. LMP2/ $\beta 1 \mathrm{i}$ is a potential diagnostic-biomarker under the combination of candidate molecules, for instance cyclin B1, cyclin E and calponin h1 and ki-67/MIB1 counts for uterine mesenchymal tumors, especially human uterine LMS, and may be a targeted-molecule for a new therapeutic approach.
\end{abstract}

Keywords: LMP2/ß1i; Uterine Leiomyosarcoma; Uterine Leiomyoma; Biomarker

\section{Introduction}

The uterus, the organ in which the embryo grows, is composed of three layers, the uterine endometrium which serves as a bed for the embryo; the myometrium of the wall which protects the embryo; and a serous membrane enveloping the uterus. In general, the term uterine tumor refers to an epithelial malignant tumor of the uterus, which is roughly classified as a tumor of the uterine cervix or the uterine body. Because of the prevalence of screening, uterine cervix cancer is decreasing in incidence, and usually detected at a very early stage. In contrast, cancer of the uterine body is increasing in incidence, and rarely detected at the initial stages. While most tumors of the uterine body are adenocarcinomas (derived from the subintimal gland), tumors of the uterine cervix are classified into squamous cancer and adenocarcinoma. Smooth muscle tumors (SMTs) which develop in the myometrium have been traditionally divided into benign uterine leiomyoma (LMA) and malignant uterine leiomyosarcoma (LMS) based on cytological atypia, mitotic activity and other criteria. Uterine LMS is relatively rare, having an estimated annual incidence of 0.64 per 100,000 women [1]. Uterine LMS accounts for $2 \%$ to $5 \%$ of tumors of the uterine body and develops more often in the muscle layer of the uterine body than in the uterine cervix. As uterine LMS is resistant to chemotherapy and radiotherapy, surgical intervention is virtually the only means of clinical treatment [2-4]. The prognosis for uterine LMS is not good, and the five-year survival rate is approximately $35 \%$ [5]. However, developing an efficient adjuvant therapy is expected to improve survival rate. Uterine LMA may occur in as many as 70\% 80\% of women by the age of 50 years [6]. Distinguishing uterine LMA from uterine LMS is very difficult, and a diagnosis generally requires surgery and cytoscopy [7]. 
Diagnostic categories for uterine SMTs and morphological criteria are used to assign cases [8,9] (Attention 1). The non-standard subtypes of uterine SMTs such as the epithelioid and myxoid types are classified in a different way using these features, so the establishment of a diagnostic method for the identification of non-standard smooth muscle differentiation is important [8,9].

High estrogen levels are considered to significantly influence the development of tumors in the uterine body [10-12]. The mechanisms by which uterine LMA and uterine LMS develop are not yet known, though tumors that have developed in the myometrium for some reason gradually become larger due to the influence of the female hormone and generate tumors. However, no correlation between the development of uterine LMS and hormonal conditions, and no obvious risk factors has been found. Although cases accompanied by hypocalcaemia or eosinophilia have been reported, neither clinical abnormality is an initial risk factor for uterine LMS. The identification of a risk factor associated with the development of uterine LMS would significantly contribute to the development of preventive and therapeutic treatments.

\section{Spontaneous Development of Uterine Leiomyosarcoma in LMP2/ק1i-Deficient Mice}

Cytoplasmic proteins are mostly degraded by a protease complex, which has many substrates consisting of twenty-eight 20 to $30-\mathrm{kDa}$ subunits, referred to as the $20 \mathrm{~S}$ proteasome $[13,14]$. The proteasomal degradation is essential for many cellular processes, including the cell cycle, the gene expression and immunological function [15]. Interferon (IFN)- $\gamma$ induces the expression of large numbers of responsive genes, proteasome subunits, i.e., low-molecular mass polypeptide (LMP)2/ $/ 1 \mathrm{i}, \mathrm{LMP} 7 / \beta 5 \mathrm{i}$, and LMP10/ $\beta 2 \mathrm{i}[16]$ (Figure 1).

The individual expression of LMP $2 / \beta 1 \mathrm{i}, \mathrm{LMP} 7 / \beta 5 \mathrm{i}$ and LMP10/ $\beta 2 \mathrm{i}$ subunits in various cell types or tissues is believed to contribute to the initiation and development of disorders. A recent study revealed a unique role for LMP7/ $\beta 5 \mathrm{i}$ in controlling pathogenic immune responses and provided a therapeutic rationale for targeting LMP7/ $\beta 5$ i in autoimmune disorders, especially rheumatoid arthritis [17].

Homozygous mice deficient in LMP2/ $\beta 1$ i show tissueand substrate-dependent abnormalities in the biological functions of the proteasome [18]. Here we identify LMP $2 / \beta 1 \mathrm{i}$, as obligatory for tumor surveillance and demonstrate a tissue-specific role for LMP $2 / \beta 1 \mathrm{i}$ in protection from spontaneous uterus neoplasms. In short, uterine LMS reportedly occurred in female LMP2/ $\beta 1$ ideficient mice at age 6 months or older, and the incidence at 14 months of age was about 40\% [19,20] (Fig-

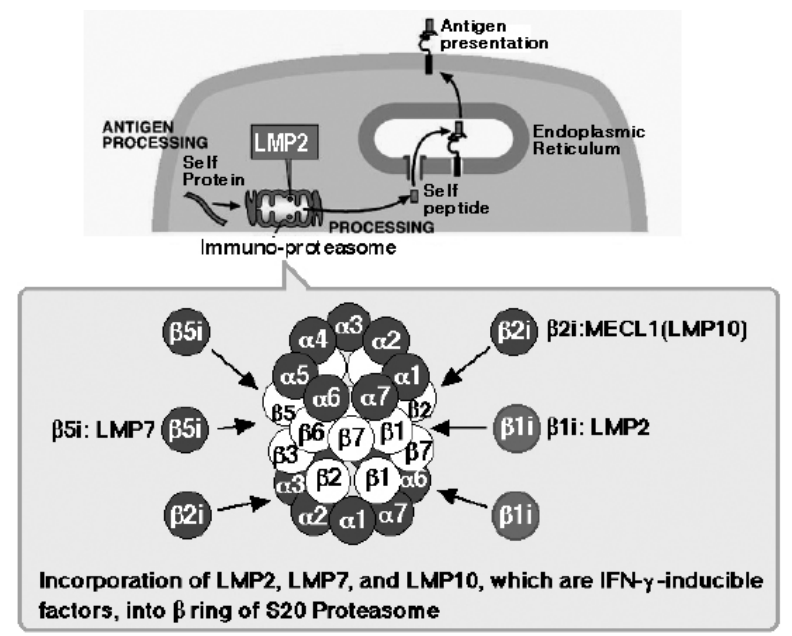

Figure 1. The immuno-proteasomal degradation pathway is essential for antigen presentation by MHC class I.

ure 2). The curve indicating the incidence in mice is similar to that indicating the incidence of human uterine LMS, which occurs after menopause. Histological studies of LMP2/ $\beta 1$ i-lacking uterine tumors have revealed characteristic abnormalities of uterine LMS, and the tumors lacked lymphoid infiltrates, a sign of immune recognition, and consisted of uniform elongated myo- metrium cells arranged into bundles [19] (Figure 2).

The nuclei of the tumor cells varied in size and shape, furthermore, mitosis was frequent. In contrast, the myometrium cells of its parental mice, C57BL/6 mice were normal in appearance. Whereas relatively few ki-67/ MIB1-positive cells, the proliferating cells of solid tumors, were observed in the basal cell layer of the normal myometrium, most of the basal cells vividly expressed ki-67/MIB1 in LMP2/ß1i-deficient mice [19] (Figure 2). Immunohistochemistry (IHC) study indicates abnormal proliferation of the LMP2/ $\beta 1$ i-lacking cells in the basal layer. LMP $2 / \beta 1$ i-deficient mice that have developed uterine LMS undergo considerable weight loss, and then die by 14 months of age $[19,20]$. The LMP2/ $\beta 1$ i-deficient mice also exhibit skeletal muscle metastasis from uterine LMS. Therefore it is likely that LMP $/ / \beta 1$ i-deficient mice with uterine LMS die as a result of the tumor mass and metastasis $[19,20]$. In general, it is not easy to distinguish uterine LMA from uterine LMS, however, in mice, because of such characteristic pathological findings, significant weight loss, and skeletal muscle metastasis, a tumor that develops in the uterus of an LMP2/ $\beta 1 \mathrm{i}$-deficient mouse can be considered malignant, i.e., uterine LMS [19,20].

\section{Defective LMP2/ß1i Expression in Human Leiomyosarcoma}

IHC studies were performed to demonstrate the validity and reliability of $\mathrm{LMP} 2 / \beta 1 \mathrm{i}$ as a diagnostic biomarker 


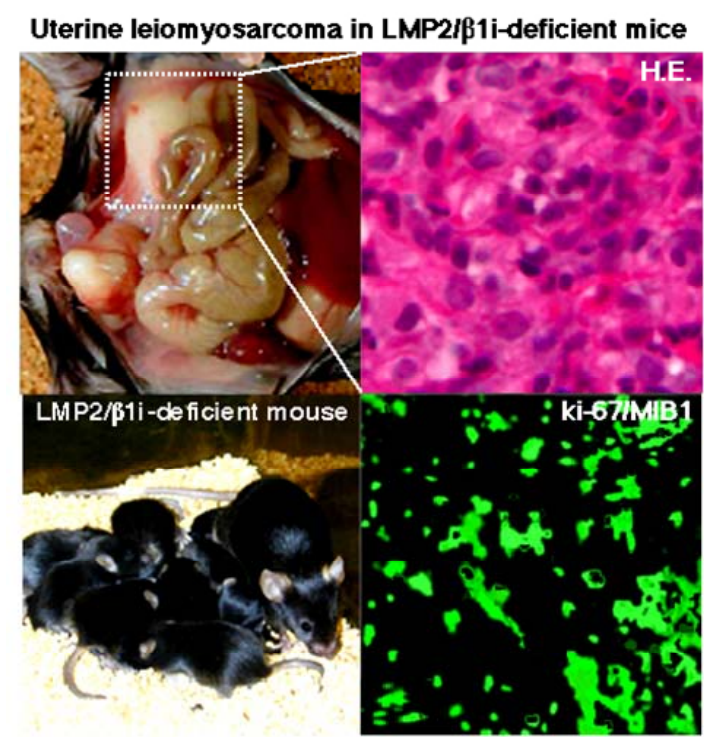

Figure 2. Homozygous mice deficient in LMP2/ß1i, an IFN-yinducible factor, show tissue- and substrate-dependent abnormalities in the biological functions of the proteasome. uterine LMS reportedly occurred in female LMP2/ $\beta 1 i$ deficient mice at age 6 months or older, and the incidence at 14 months of age was about $40 \%$.

under the combination of other candidate molecules, for instance cyclin B1, cyclin E and calponin h1, which reportedly function as anti-tumorigenic factor in human uterine LMS [21-25]. IHC experiments revealed a serious loss in the ability to induce LMP2/ $/ 31 \mathrm{i}$ and calponin h1 expression in human uterine LMS tissue in comparison with uterine LMA or normal myometrium located in the same section [21-25]. Of the 54 cases we examined with uterine LMS, 46 were negative for LMP2/ $\beta 1$ i expression, 4 were focally positive, and 2 were partially positive $[21,22,24]$. Two uterine LMS cases were stained

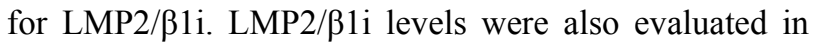
skeletal muscle and rectum metastases from individual uterine LMS patients. Pathological examination of surgical samples showed the presence of a mass measuring $3 \mathrm{~cm}$ in its largest diameter in the lumbar quadrate muscle without a fibrous capsule. All lymph nodes were negative for uterine LMS metastases, and IHC analyses showed positivity for ki-67/MIB1 and negativity for LMP2/ß1i. Histological findings were consistent with metastatic LMS for the skeletal muscle and rectum lesions. In western blotting and RT-PCR experiments, LMP2/ $\beta 1$ i was expressed in normal myometrium, but not in human uterine LMS, both strongly supportive of the IHC findings [21,22]. Although we has previously demonstrated that the abnormal expression of the ovarian steroid receptors, TP53 and ki-67/MIB1 and mutations of TP53 were frequently associated with uterine LMS, defective LMP2/ $/ 1$ i expression appears to be more characteristic of uterine LMS than these factors [21-25].

\section{LMP2/ß1i Differential Expressions in Human Uterine Mesenchymal Tumors}

In the case of gynecological cancers, such as breast cancer, a female hormonal imbalance is often a risk factor for developing tumors [10-12]. As in the case of uterine LMA, however, a correlation between the development of uterine LMS, the female hormone, and hormone receptors has yet to be elucidated. A recent report showed the expression of Lmp 2 mRNA and protein in luminal and glandular epitheliua, placenta villi, trophoblastic shells, and arterial endothelial cells [26-27]. These results implicate LMP2/B1i in the invasion of placental villi, degradation of the extracellular matrix, immune tolerance, glandular secretion, and angiogenesis $[26,27]$. The present study should help to elucidate the regulatory role of LMP2/ $\beta 1 \mathrm{i}$ in the implantation of embryos [26-29]. Unfortunately, it is unclear whether defective LMP2/ $/ 31 \mathrm{i}$ expression is involved in the onset of uterine LMS. Risk factors for its development however, have not been identified because of the absence of a suitable animal model. The LMP2/ק1i-deficient mouse was the first animal model of spontaneous uterine LMS to be established $[19,20]$. Defective LMP2/ $\beta 1$ i expression may be one of the causes of uterine LMS. To demonstrate whether LMP2/ $\beta 1 \mathrm{i}$ is a potential biomarker for distinguishing uterine LMS from uterine LMA, we are investigating the reliability and characteristics of LMP $2 / \beta 1 \mathrm{i}$ as a diagnostic indicator with several clinical research facilities. To demonstrate whether LMP2/ $\beta 1 \mathrm{i}$ is a potential biomarker for distinguishing human uterine LMS from uterine LMA under the combination with other candidate molecules, especially cyclin B1, cyclin E calponin h1, which are identified as potential diagnostic candidates, and ki-67/ MIB1 counts [21,22,24,25,30-37], we are investigating the reliability and characteristics of LMP $2 / \beta 1 \mathrm{i}$ as a diagnostic indicator with several clinical research facilities [22-25,30-32]. The clinical research is yet to be concluded, and large-scale clinical studies need to be performed with additional clinical research facilities. Histologic and IHC characteristics of uterine mesenchymal tumors including mitotically active leiomyoma, bizarre leiomyoma, lipoleiomyoma, uterine smooth muscle tumors of uncertain malignant potential (STUMP), leiomyomatoid angiomatous neuroendocrin tumor (LANT) are summarized [32-37]. Clarification of the correlation between these factors and the development of human uterine LMS and the identification of specific risk factors may lead to the development of new treatments for the disease. Uterine LMS is refractory to chemotherapy and has a poor prognosis. The molecular biological and cytological information obtained from LMP2/ $\beta 1$ i-deficient mice will contribute remarkably to the development of preventive methods, a potential diagnostic-biomarker, and new therapeutic approaches against human uterine 
LMS.

\section{Final Considerations}

Human uterine LMS is refractory to chemotherapy and has a poor prognosis. Defective LMP $2 / \beta 1 \mathrm{i}$ expression is likely to be one of the risk factors in the development of human uterine LMS as it is in the LMP2/ $\beta 1$ i-deficient mouse. LMP2/ $\beta 1$ i might function as an anti-tumorgenic factor in human uterine LMS. The molecular biological and cytological information obtained from LMP2/ $\beta 1$ ideficient mice will contribute remarkably to the development of preventive methods, a potential diagnostic biomarker, and new therapeutic approaches against human mesenchymal tumors, especially human uterine LMS.

\section{Acknowledgements}

We sincerely thank Professor Luc Van Kaer (Vanderbilt University Medical Center). This study was supported in part by grants from the Ministry of Education, Culture, Science and Technology, and The Foundation of Osaka Cancer Research, The Ichiro Kanehara Foundation for the Promotion of Medical Science and Medical Care, The foundation for the Promotion of Cancer Research, The Kanzawa Medical Research Foundation, The Shinshu Medical Foundation, and The Takeda Foundation for Medical Science.

\section{REFERENCES}

[1] C. Zaloudek and M. R. Hendrickson, "Mesenchymal tumors of the uterus, in R. J. Kurman (ed): Blaustein's Pathology of the Female Genital Tract (ed 5)," New York, Springer-Verlag, Vol. 5, 2002, pp. 561-578.

[2] T. I. Wu, T. C. Chang, S. Hsueh, K. H. Hsu, H. H. Chou, H. J. Huang and C. H. Lai, "Prognostic Factors and Impact of Adjuvant Chemotherapy for Uterine Leiomyosarcoma," Gynecologic Oncology, Vol. 100, No. 1, 2006 , pp.166-172.doi: /10.1016/j.ygyno.2005.08.010

[3] M. M. Leitao, R. A. Soslow, D. Nonaka, A. B. Olshen, C. Aghajanian, P. Sabbatini, J. Dupont, M. Hensley, Y. Sonoda, R. R. Barakat and S. Anderson, "Tissue Microarray Immunohisto Chemical Expression of Estrogen, Progesterone, and Androgen Receptors in Uterine Leiomyomata and Leiomyosarcoma," Cancer, Vol. 101, No. 6, 2004, pp. $1455-1462$. doi:10.1002/cncr.20521

[4] E. A. Perez, L. Pusztai and M. Van de Vijver, "Improving Patient Care through Molecular Diagnostics," Seminars in Oncology, Vol. 31, No. 10, 2004, pp. 14-20. doi:10.1053/j.seminoncol.2004.07.017

[5] http://www.cancer.gov/cancertopics/pdq/treatment/uterine sarcoma/HealthProfessional/page1\#Section_87

[6] http://cancer.gov/cancertopics/pdq/treatment/uterine sarcoma/HealthProfessional
[7] H. L. Evans, S. P. Chawla, C. Simpson and K. P. Finn, "Smooth Muscle Neoplasms of the Uterus Other than Ordinary Leiomyoma," A Study of 46 Cases, with Emphasis on Diagnostic Criteria and Prognostic Factors, Cancer, Vol. 62, No. 10, 1988, pp. 2239-2247. doi:10.1002/1097-0142(19881115)62:10<2239::AID-CN CR2820621028>3.0.CO;2-Y

[8] R. J. Kurma, "Pathology of the Female Genital Tract," 4th edition, Springer-Verlag; New York, Vol. 4, 2001, p. 499.

[9] Diagnostic Criteria for LMS, Adapted from 2003 WHO Guidelines: World Health Organization Classification of Tumours: Pathology and Genetics, Pathology and Genetics of Tumours of the Breast and Female Genital Organs, IARC Press, France, 2003.

[10] J. F. Lin and B. M. Slomovitz, "Uterine Sarcoma," Current Oncology Reports, Vol. 10, No. 6, 2008, pp. 512-518. doi:10.1007/s11912-008-0077-9

[11] F. Amant, A. Coosemans, M. Debiec-Rychter, D. Timmerman and I. Vergote, "Clinical Management of Uterine Sarcomas," The Lancet Oncology, Vol. 10, No. 12, 2009, pp. 1188-1198.

doi:10.1016/S1470-2045(09)70226-8

[12] M. Miettinen and J. F. Fetsch, "Evaluation of Biological Potential of Smooth Muscle Tumours," Histopathology, Vol. 48, No. 1, 2006, pp. 97-105. doi:10.1111/j.1365-2559.2005.02292.x

[13] J. M. Peters, W. W. Franke and J. A. Kleinschmidt, Distinct $19 \mathrm{~S}$ and $20 \mathrm{~S}$ Subcomplexes of the $26 \mathrm{~S}$ Proteasome and Their Distribution in the Nucleus and the Cytoplasm," Journal of Biological Chemistry, Vol. 269, 1994, pp.7709-7718.

[14] H. Lodish, A. Berk, P. Matsudaira, C. A. Kaiser, M. Krieger, M. P. Scott, S. L. Zipursky and J. Darnell, 2004 "3", Mol Cell Biol (5th ed.), New York: W. H. Freeman and CO. Vol. 5, 2004, pp. 66-72.

[15] I. M. Konstantinova, A. S. Tsimokha and A. G. Mittenberg, "Role of Proteasomes in Cellular Regulation," International Review Cell and Molecular Biology, Vol. 267, 2008, pp. 59-124. doi:10.1016/S1937-6448(08)00602-3

[16] J. Wang and M. A. Maldonado, "The Ubiquitin-Proteaso me System and Its Role in Inflammatory and Autoimmune Diseases," Cell and Molecular Immunology, Vol. 3, 2006, pp. 255-261.

[17] T. Muchamuel, M. Basler, M. A. Aujay, E. Suzuki, K. W. Kalim, C. Lauer, C. Sylvain, E. R. Ring, J. Shields, J. Jiang, P. Shwonek, F. Parlati, S. D. Demo, M. K. Bennett, C. J. Kirk and M. Groettrup, "A Selective Inhibitor of the Immunoproteasome Subunit LMP7 Blocks Cytokine Production and Attenuates Progression of Experimental Arthritis," Nature Medicine, Vol. 15, 2009, pp.781-788. doi:10.1038/nm.1978

[18] L. Van Kaer, P. G. Ashton-Rickardt, M. Eichelberger, M. Gaczynska, K. Nagashima, K. L. Rock, A. L. Goldberg, P. C. Doherty and S. Tonegawa, "Altered Peptidase and Viral-specifi c T Cell Response in LMP2 Mutant Mice," Immunity, Vol. 1, 1994, pp. 533-541. 
[19] T. Hayashi and D. L. Faustman, "Development of Spontaneous Uterine Tumors in Low Molecular Mass Polypeptide-2 Knockout Mice," Cancer Research, Vol. 62, 2002, pp. 24-27.

http://cancerres.aacrjournals.org/content/62/1/24

[20] T. Hayashi, A. Horiuchi, K. Sano, N. Hiraoka, Y. Kanai, T. Shiozawa, S. Tonegawa and I. Konishi, "Molecular Approach on Uterine Leiomyosarcoma: LMP2-deficient Mice as an Animal Model of Spontaneous Uterine Leiomyosarcoma," Sarcoma 2011: 476498, Epub 2011 Mar 8, http://www.hindawi.com/journals/srcm/2011/476498/

[21] T. Hayashi, Y. Kobayashi, S. Kohsaka and K. Sano, "Mutation in the ATP-binding Region of JAK1, Identified in Human Uterine Leiomyosarcomas, Results in Defective Interferon-gamma Inducibility of TAP1 and LMP2," Oncogene, Vol. 25, 2006, pp. 4016-4026. doi:10.1038/sj.onc.1209434

[22] T. Hayashi, A. Horiuchi, K. Sano, N. Hiraoka, M. Kasai, T. Ichimura, S. Nagase, O. Ishiko, Y. Kanai, N. Yaegashi, H. Aburatani, T. Shiozawa, S. Tonegawa and I. Konishi, "Potential Role of LMP2 as Tumor-suppressor Defines New Targets for Uterine Leiomyosarcoma Therapy," Scientific Reports, Vol. 1, 2011, p. 180. doi:10.1038/srep00180

[23] Y. L. Zhai, Y. Kobayashi, A. Mori, A. Orii, T. Nikaido, I. Konishi and S. Fujii, "Expression of Steroid Receptors, $\mathrm{Ki}-67$, and p53 in Uterine Leiomyosarcomas," International Journal Gynecological Pathology, Vol. 18, No. 1,2004, pp. 20-28. doi:10.1097/00004347-199901000-00004

[24] T. Hayashi, A. Horiuchi, K. Sano, N. Hiraoka, M. Kasai, T. Ichimura, S. Nagase, O. Ishiko, Y. Kanai, N. Yaegashi, H. Aburatani, T. Shiozawa, S. Tonegawa and I. Konishi, "Potential role of LMP2 as an Anti-oncogenic Factor in Human Uterine Leiomyosarcoma: Morphological Significance of Calponin h1," FEBS Letter, Vol. 586, 2012, pp. 1824-1831.doi:10.1016/j.febslet.2012.05.029

[25] T. Hayashi, A. Horiuchi, K. Sano, N. Hiraoka, T. Ichimura, T. Sudo, O. Ishiko, N. Yaegashi, H. Aburatani and I. Konishi, "Potential Diagnostic Biomarkers for Human Uterine Mesenchymal Tumors: Especially LMP2/ $\beta 1 \mathrm{i}$ and Cyclin B1-differential Expression," Oncology Letters, 2013.

[26] H. X. Wang, H. M. Wang, Q. L. Li and P. L. Judoson, "Expression of Proteasome Subunits Low Molecular Mass Polypeptide (LMP) 2 and LMP7 in the Endometrium and Placenta of Rhesus Monkey (Macaca mulatta) during early Pregnancy," Biology of Reproduction, Vol. 71, No. 4, 2004, pp. 1317-1324. doi:10.1095/biolreprod.104.030213

[27] H. X. Wang, H. M. Wang, H. Y. Lin, Q. Yang, H. Zhang, B. K.Tsang and C. Zhu, "Proteasome Subunit LMP2 is Required for Matrix Metalloproteinase-2 and -9 expression and Activities in Human Invasive Extravillous Trophoblast Cell Line," Journal of Cellular Physiology, Vol. 206, No. 3, pp. 616-623. doi:10.1002/jep.20508

[28] J. J. Fu, P. Lin, X. Y. Lv, X. J. Yan, H. X. Wang, C. Zhu, B. K. Tsang, X. G. Yu and H. Wang, "Low Molecular
Mass Polypeptide-2 in Human Trophoblast: Over-expression in Hydatidiform Moles and Possible Role in Trophoblast Cell Invasion," Placenta, Vol. 30, No. 4, 2009, pp. 305-312. doi:10.1016/j.placenta.2009.01.005

[29] T. Hayashi, A. Horiuchi, H. Aburatani, N. Yaegashi, S. Tonegawa and I. Konishi, "A Potential Diagnostic Biomarker: Proteasome LMP2/ $\beta 1$ i-differential Expression in Human Uterus Neoplasm," Nature Proceedings 2012, March 2: hdl:10101/npre.2012.7082.1.

[30] A. Horiuchi, T. Nikaido, K. Ito, Y. Zhai, A. Orii, S. Taniguchi, T. Toki and S. Fujii, "Reduced Expression of Calponin h1 in Leiomyosarcoma of the Uterus," Lab Investment, Vol. 78, 1998, pp. 839-846. http://www.ncbi.nlm.nih.gov/pubmed/9690561

[31] A. Horiuchi, T. Nikaido, S. Taniguchi and S. Fujii, "Possible Role of Calponin h1 as a Tumor Suppressor in Human Uterine Leiomyosarcoma," Journal of the National Cancer Institute, Vol. 91, No. 9, 1999, pp.790-796. doi:10.1093/jnci/91.9.790

[32] Y. L. Zhai, T. Nikaido, T. Shiozawa, A. Orii and S. Fujii, "Expression of Cyclins and Cyclin-dependent Kinases in Smooth Muscle Tumors of the Uterus," International Journal of Cancer, Vol. 84, 1999, pp. 224-250.

[33] P. P. Ip, A. N. Cheung and P. B. Clement, "Uterine Smooth Muscle Tumors of Uncertain Malignant Potential (STUMP): A Clinicopathologic Analysis of 16 Cases," The American Journal of Surgical Pathology, Vol. 33, 2009, pp. 992-1005.

doi:10.1097/PAS.0b013e3181a02d1c

[34] P. P. Ip, K. Y. Tse and K. F. Tam, "Uterine Smooth Muscle Tumors Other than the Ordinary Leiomyomas and LeioMyosarcomas: A Review of Selected Variants with Emphasis on Recent Advances and Unusual Morphology that may Cause Concern for Malignancy," Advances in Anatomic Pathology, Vol. 17, 2010, pp. 91-112.

doi:10.1097/PAP.0b013e3181cfb901

[35] I. Vajtai, R. Sahli, A. Kappeler, E. R. Christ and R. W. Seiler, "Leiomyomatoid Angiomatous Neuroendocrine Tumor (LANT) of the Pituitary: A Distinctive Biphasic Neoplasm with Primitive Secretory Phenotype and Smooth Muscle-rich Stroma," Acta Neuropathologica, Vol. 111, No. 4, 2006, pp. 397-399.

doi:10.1007/s00401-006-0065-9

[36] N. Sakashita, M. Yamada, T. Nakagawa, H. Yamasaki and M. Takeya, "A Leiomyomatoid Angiomatous Neuroendocrine tumor of the Myometrium: Case Study with Ultrastructural Analysis," Human Pathology, Vol. 39, No. 5, 2008, pp. 788-792. doi:10.1016/j.humpath.2007.09.017

[37] R. Avritscher, R. B. Iyer1, J. Ro and G. Whitman, "LipoleioMyoma of the Uterus," American Journal of Radiology, Vol. 177, 2001, p. 856. 


\section{Attention 1}

The typical gross appearance is a large $(>10 \mathrm{~cm})$, poorly circumscribed mass with a soft, fleshy consistency and a variegated cut surface that is grey-yellow to pink, with foci of hemorrhage and necrosis [8,9]. The histologic classification of uterine sarcomas is based upon homology to normal cell types and include uterine LMS (analogous to myometrium), stromal sarcoma (analogous to endometrial stroma), and other heterologous cell types (i.e., osteosarcoma, liposarcoma). Microscopically, most human uterine LMS are overtly malignant, with hypercellularity, coagulative tumor cell necrosis, abundant mitoses [ $>10$ to 20 mitotic figures (mf) per 10 high power fields (hpf)], atypical mitoses, cytologic atypia, and infiltrative borders. Mitotic rate is the most important determinant of malignancy, but is modified by the presence of necrosis and cytologic atypia. The diagnosis of human uterine LMS may be made in the presence of tumor necrosis and any mitoses. In the absence of tumor necrosis, the diagnosis can be made with moderate to severe cytologic atypia and a mitotic index greater than $10 \mathrm{mf} / 10 \mathrm{hpf}$. Without tumor necrosis and significant atypia, a high mitotic index is compatible with a benign clinical course; however, data is limited [8,9]. 